\title{
Predictors of Heart Failure Caused by Volume Overload Using an Irrigation Catheter during Catheter Ablation for Atrial Fibrillation
}

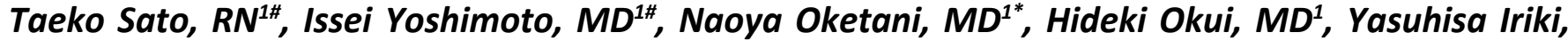 $M D^{1}$, Hitoshi Ichiki, MD', Ryuichi Maenosono, $P h D^{2}$, Fuminori Namino, $\mathbf{M T}^{2}$, Masaaki Miyata, $\mathrm{MD}^{1}$ and Mitsuru Ohishi, MD ${ }^{1}$}

\author{
${ }^{1}$ Department of Cardiovascular Medicine and Hypertension, Graduate School of Medical and \\ Dental Sciences, Kagoshima University, Japan \\ ${ }^{2}$ Clinical Laboratory Unit, Kagoshima University Hospital, Japan \\ ${ }^{\#}$ These authors contributed equally to this work.
}

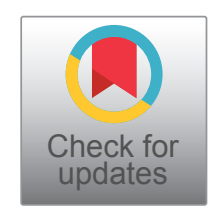

*Corresponding author: Naoya Oketani, MD, Department of Cardiovascular Medicine and Hypertension, Graduate school of Medical and Dental Sciences, Kagoshima University, 8-35-1 Sakuragaoka, Kagoshima 890-8520, Japan, Tel: +81-99275-5318, Fax: +81-99-265-8447

\begin{abstract}
Background: Predictors of heart failure caused by volume overload are unclear. This study to investigate the predictors of heart failure caused by volume overload using an irrigation catheter during atrial fibrillation (AF) ablation.

Methods: This study included 431 consecutive patients with $\mathrm{AF}$ who underwent ablation in their first session from April 2009 to December 2015. According to right ventricular systolic pressure (RVSP) after AF ablation, patients were divided into high ( $\geq 40 \mathrm{mmHg}, \mathrm{n}=62$ ) or normal RVSP $(<40$ $\mathrm{mmHg}, \mathrm{n}=312$ ) groups.

Results: There were significant differences in the rates of age $\geq 65$-years-old ( $53.2 \%$ vs. $32.1 \%$ ), history of congestive heart failure $(16.1 \%$ vs. $8.0 \%)$, hypertension $(67.7 \%$ vs. $50 \%)$, and diabetes mellitus $(25.8 \%$ vs. $6.4 \%)$, as well as serum creatinine concentrations (0.92 vs. $0.83 \mathrm{mg} / \mathrm{dl})$, B-type natriuretic peptide levels (165.9 vs. $109.0 \mathrm{pg} / \mathrm{ml}$ ), left atrial dimension (44.4 vs. $41.0 \mathrm{~mm}$ ), mitral inflow $\mathrm{E}$ wave velocity before ablation ( $E$ wave, 76.6 vs. $70.2 \mathrm{~cm} / \mathrm{sec}$ ), and the total amount of injection (3591 vs. $3282 \mathrm{ml}$ ) between the high and normal RVSP groups. There were no significant differences in sex, body mass index, left ventricular ejection fraction, and radiofrequency duration. Age $\geq 65$-years-old ( $p$ $=0.004)$, history of diabetes mellitus $(p<0.001)$, left atrial dimension $(p=0.021)$, and $E$ wave $(p=0.049)$ showed significant differences in multiple analysis between the high and normal RVSP groups.

Conclusions: Close observation is mandatory with an older age, greater left atrial dimension and mitral E wave, and/or a history of diabetes mellitus to avoid heart failure after AF ablation using irrigation catheters.
\end{abstract}

\section{Keywords}

Ablation, Atrial fibrillation, Irrigation catheter, Volume overload

\section{Introduction}

Catheter ablation is an effective approach for managing patients with atrial fibrillation (AF) [1-7]. Currently, irrigation catheters are widely used in AF ablation. This causes volume overload during the procedure and occasionally causes heart failure after the procedure. Volume overload during catheter ablation caused heart failure not only in the patient with reduced ejection fraction but also who with preserved left ventricular ejection fraction. Heart failure with preserved left ventricular ejection fraction is a widely accepted disease [810], but there have been few reports of the predictors of heart failure induced by volume overload in clinical practice. Therefore, we investigated the predictors of heart failure caused by volume overload during catheter ablation for AF using an irrigation catheter.

\section{Methods}

\section{Study population and protocol}

This study comprised 431 consecutive patients (112 females, 319 males, mean age of $60 \pm 10$ years). We

Citation: Sato T, Yoshimoto I, Oketani N, Okui H, Iriki Y, et al. (2019) Predictors of Heart Failure Caused by Volume Overload Using an Irrigation Catheter during Catheter Ablation for Atrial Fibrillation. Int J Clin Cardiol 6:155. doi.org/10.23937/2378-2951/1410155

Accepted: August 12, 2019; Published: August 14, 2019

Copyright: (C) 2019 Sato T, et al. This is an open-access article distributed under the terms of the Creative Commons Attribution License, which permits unrestricted use, distribution, and reproduction in any medium, provided the original author and source are credited. 
included 248 (58\%) patients with paroxysmal AF and 183 (42\%) patients with persistent/long-standing persistent AF with symptomatic drug refractory AF. These patients successfully underwent complex fractionated atrial electrogram (CFAE)-guided ablation combined with ( $\mathrm{n}=$ 137 ) or without ( $n=288$ ) pulmonary vein isolation from April 2009 to December 2015. In this study, according to right ventricular systolic pressure (RVSP) estimated using echocardiography after AF ablation, patients were divided into high ( $=40 \mathrm{mmHg}, \mathrm{n}=62$ ) or normal RVSP $(<40 \mathrm{mmHg}, \mathrm{n}=312$ ) groups. RVSP is one of the most valuable surrogate markers for heart failure and the normal range was below $40 \mathrm{mmHg}$. We excluded 57 patients whose echocardiographic data before ablation or the day after ablation were not available $(n=39)$, or RVSP calculated by echocardiography before ablation was $\geq 40 \mathrm{mmHg}$ before ablation $(n=18)$.

All antiarrhythmic drugs were discontinued at least five half-lives before ablation, with the exception of amiodarone, which was discontinued at least 3 months before ablation. Warfarin, rivaroxaban, or edoxaban was not discontinued, and dabigatran or apixaban was discontinued only on the morning of the procedure. All of the patients provided written informed consent for the procedure. This study protocol was approved by our institutional ethics committee. AF was defined in accordance with the 2007 Heart Rhythm Society Expert Consensus Statement [11]. Paroxysmal AF was defined as recurrent $\mathrm{AF}$ ( $\geq 2$ episodes) that terminated spontaneously within 7 days. Persistent AF was defined as AF that was sustained for longer than 7 days or as AF lasting less than 7 days, but necessitating pharmacological or electrical cardioversion. AF lasting longer than 1 year was defined as long-standing persistent AF.

\section{Mapping and ablation of $\mathrm{AF}$}

The AF ablation technique, which was described by Nademanee, et al. $[7,12,13]$, was used for this study, as previously reported [14-17]. In brief, a 3.5-mm NaviStar ThermoCool catheter (DF curve; Biosense Webster, Diamond Bar, CA, USA) was used in all cases, and the irrigation flow was $30 \mathrm{ml} / \mathrm{min}$. After the coronary sinus (CS) was cannulated via the femoral vein by a decapolar catheter (Biosense Webster) for recording and induction, patients underwent non-fluoroscopic electroanatomical mapping with CARTO3 (Biosense Webster). Heparin (5000 IU for the initial bolus, 1000-2000 IU for subsequent boluses as required to maintain an activated clotting time $>300$ s) was administered for anticoagulation.

All electroanatomical maps were created for patients who were in AF, which occurred spontaneously or by induction. Burst pacing using a CS catheter to a lower limit of 1:1 capture or up to $150 \mathrm{~ms}$ was used to induce AF occasionally with a $0.01-0.02 \mu \mathrm{g} / \mathrm{kg} / \mathrm{min}$ isoproterenol infusion. When AF was terminated during the ablation procedure in patients with paroxysmal AF,
AF was re-induced until it was no longer inducible because paroxysmal AF could be terminated spontaneously. Electroanatomical maps were created and displayed as a shortest complex interval map, and the areas of the CFAE were also identified manually, tagged, and associated with the atrial anatomy created by CARTO3. This enabled the operators to associate areas of the CFAE with the left atrium (LA), CS, and occasionally the right atrium, thereby identifying target sites for ablation.

Bipolar recordings were filtered at $30-500 \mathrm{~Hz}$ and the CFAE was defined as follows: 1) fractionated electrograms composed of two or more deflections and/or a perturbation of the baseline with continuous deflection of a prolonged activation complex; and 2) atrial electrograms with a short cycle length ( $\leq 120 \mathrm{~ms})$.

After acquiring the shortest complex interval map associated with the CFAE, we searched the areas of CFAEs by referring to the tagged points because these areas have temporal spatial stability. Radiofrequency applications were delivered with a maximal power of $40 \mathrm{~W}$ with irrigation rates of $30 \mathrm{ml} / \mathrm{min}(3.5-\mathrm{mm}$ NaviStar ThermoCool catheter; Biosense Webster). The power was reduced to $15 \mathrm{~W}$ in the posterior LA closed to the esophagus or in the CS. The primary endpoints during RF ablation were either complete elimination of areas of the CFAE or conversion of AF to sinus rhythm occasionally with injection of nifekalant $(0.3$ $\mathrm{mg} / \mathrm{kg}$ intravenously over $10 \mathrm{~min}$, maximum of twice). Nifekalant is a Class III antiarrhythmic drug similar to ibutilide, which is not available in Japan. If the atrial arrhythmias were not successfully terminated, internal cardioversion was performed.

\section{Possible predictors}

The patients' background, blood analysis before ablation, echocardiographic data before ablation, medications, some data in AF ablation, such as the volume of injection, and the procedure time were analyzed.

\section{Statistical analysis}

According to RVSP after AF ablation, patients were divided into high ( $\geq 40 \mathrm{mmHg}, \mathrm{n}=62$ ) or normal RVSP $(<40 \mathrm{mmHg}, \mathrm{n}=312$ ) groups. All continuous data are presented as the mean value \pm standard deviation. Data were analyzed using the unpaired Student's t-test in equal variance or Welch's t-test in unequal variance. Disease prevalence, sex, and the proportion of patients taking medications were compared between the two groups using Pearson's chi-square test. Fisher's exact test was used to determine statistical significance in the analysis of contingency tables in which sample sizes were small. Odds ratios (ORs) were calculated by multivariate analysis with logistic regression analysis. Differences between the two groups were considered significant at a $p$ value $<0.05$. All statistical analyses were performed with JMP ${ }^{\circledR}$ Pro version 11 (SAS Institute, Inc., Cary, NC, USA) for Windows. 
Table 1: Patients characteristics.

\begin{tabular}{|c|c|c|c|}
\hline & $\begin{array}{l}\text { High RVSP } \\
(n=62)\end{array}$ & $\begin{array}{l}\text { Normal RVSP } \\
(n=312)\end{array}$ & $p$ value \\
\hline age (years old) & $65.4 \pm 8.4$ & $59.3 \pm 10.7$ & $<0.001$ \\
\hline gender (female) [n (\%)] & $14(22.5)$ & $87(27.9)$ & 0.39 \\
\hline body weight (kg) & $66.4 \pm 11.1$ & $65.6 \pm 11.1$ & 0.588 \\
\hline body mass index $\left(\mathrm{kg} / \mathrm{m}^{2}\right)$ & $24.7 \pm 3.8$ & $24 \pm 3.2$ & 0.141 \\
\hline paroxysmal/persistent AF [n (\%)] & $35(56.5) / 27(43.5)$ & $128(41.0) / 184(59.0)$ & 0.025 \\
\hline CHADS2 score & $1.6 \pm 1.1$ & $0.9 \pm 1.0$ & $<0.001$ \\
\hline CHA2DS2-VASc score & $2.6 \pm 1.5$ & $1.6 \pm 1.4$ & $<0.001$ \\
\hline congestive heart failure [n (\%)] & $10(16.1)$ & $25(8.0)$ & 0.045 \\
\hline hypertension [n (\%)] & $42(67.7)$ & $156(50.0)$ & 0.011 \\
\hline age $\geq 5$-years-old [n (\%)] & $33(53.2)$ & $100(32.1)$ & 0.002 \\
\hline Age $\geq 75$-years-old [n (\%)] & $8(12.9)$ & $16(5.1)$ & 0.023 \\
\hline diabetes mellitus [n (\%)] & $16(25.8)$ & $20(6.4)$ & $<0.001$ \\
\hline stroke/TIA [n (\%)] & $12(19.3)$ & $31(9.9)$ & 0.034 \\
\hline \multicolumn{4}{|l|}{ Preprocedural examination } \\
\hline mean of both CAVI & $8.49 \pm 1.37$ & $7.95 \pm 1.21$ & 0.002 \\
\hline mean of both baPWV $(\mathrm{cm} / \mathrm{sec})$ & $1658.2 \pm 306.0$ & $1509 \pm 299.6$ & 0.001 \\
\hline creatinine (mg/dl) & $0.92 \pm 0.29$ & $0.83 \pm 0.25$ & 0.012 \\
\hline eGFR $\left(\mathrm{ml} / \mathrm{min} / 1.73 \mathrm{~m}^{2}\right)$ & $61.8 \pm 15.9$ & $69.3 \pm 16.2$ & 0.001 \\
\hline hemoglobin (g/dl) & $14.3 \pm 1.6$ & $14.9 \pm 8.5$ & 0.226 \\
\hline $\mathrm{BNP}(\mathrm{pg} / \mathrm{ml})$ & $165.9 \pm 157.9$ & $109 \pm 120.7$ & 0.009 \\
\hline NT-proBNP (pg/ml) & $663.8 \pm 894.9$ & $378.3 \pm 466.5$ & 0.017 \\
\hline CRP (mg/dl) & $0.14 \pm 0.23$ & $0.10 \pm 0.25$ & 0.203 \\
\hline $\mathrm{HbA1c}(\%)$ & $5.8 \pm 0.9$ & $5.4 \pm 0.5$ & 0.006 \\
\hline \multicolumn{4}{|l|}{ Echocardiography before ablation } \\
\hline LA dimension (mm) & $44.4 \pm 6.3$ & $41.0 \pm 6.5$ & 0.001 \\
\hline LA volume (ml) & $77.2 \pm 25.7$ & $68.3 \pm 27.7$ & 0.022 \\
\hline LAVI $\left(\mathrm{ml} / \mathrm{m}^{2}\right)$ & $43.1 \pm 14.0$ & $38.4 \pm 13.3$ & 0.024 \\
\hline LV ejection fraction (\%) & $64.1 \pm 12$ & $63.8 \pm 10.2$ & 0.874 \\
\hline LV end-diastolic diameter (mm) & $47.1 \pm 4.6$ & $47.5 \pm 4.9$ & 0.512 \\
\hline LV end-systolic diameter (mm) & $30.2 \pm 6.2$ & $30.6 \pm 5.6$ & 0.585 \\
\hline LV mass index $\left(\mathrm{g} / \mathrm{m}^{2}\right)$ & $106.9 \pm 32.6$ & $101.4 \pm 30.5$ & 0.289 \\
\hline Mitral inflow E wave (cm/sec) & $76.6 \pm 22.9$ & $70.2 \pm 22.1$ & 0.039 \\
\hline deceleration time (msec) & $186.5 \pm 54.7$ & $193.3 \pm 50.5$ & 0.36 \\
\hline Peak flow velocity of TR (m/sec) & $2.3 \pm 0.2$ & $2.2 \pm 0.2$ & $<0.001$ \\
\hline $\mathrm{RVSP}(\mathrm{mmHg})$ & $31.8 \pm 4.5$ & $30.0 \pm 3.6$ & 0.004 \\
\hline IVC during exhalation (mm) & $12.4 \pm 3.3$ & $12.7 \pm 4$ & 0.513 \\
\hline IVC during inspiration (mm) & $7.2 \pm 3.0$ & $7.5 \pm 3.0$ & 0.551 \\
\hline tissue doppler septal e' wave (cm/sec) & $7.1 \pm 1.9$ & $7.9 \pm 2.5$ & 0.006 \\
\hline tissue doppler lateral e' wave (cm/sec) & $10.4 \pm 3.3$ & $10.8 \pm 3.5$ & 0.353 \\
\hline E/e' septal & $11.3 \pm 4.3$ & $9.6 \pm 4.1$ & 0.003 \\
\hline E/e' lateral & $8.0 \pm 3.7$ & $7.0 \pm 3.0$ & 0.058 \\
\hline LA appendage flow velocity calculated by TEE $(\mathrm{cm} / \mathrm{s})$ & $41.1 \pm 21.5$ & $47 \pm 20.8$ & 0.046 \\
\hline \multicolumn{4}{|l|}{ medication } \\
\hline ACE/ARB [n (\%)] & $33(53.2)$ & $113(36.2)$ & 0.012 \\
\hline beta blocker [n (\%)] & $29(46.8)$ & $126(40.4)$ & 0.351 \\
\hline class 1 antiarrhythmic drugs [n (\%)] & $15(24.2)$ & $118(37.8)$ & 0.043 \\
\hline verapamil, diltiazem [n (\%)] & $17(27.4)$ & $68(21.8)$ & 0.325 \\
\hline
\end{tabular}




\begin{tabular}{|l|l|l|l|}
\hline diuretics [n (\%)] & $16(25.8)$ & $44(14.1)$ & 0.022 \\
\hline catheter ablation & \multicolumn{3}{|l|}{} \\
\hline dose of contrast (ml) & $8.4 \pm 16.4$ & $6.9 \pm 15.8$ & 0.491 \\
\hline procedure duration (minutes) & $226.3 \pm 52.3$ & $216.5 \pm 55.1$ & 0.202 \\
\hline radiofrequency duration (minutes) & $93.7 \pm 28.4$ & $88.9 \pm 31.3$ & 0.267 \\
\hline total injection (ml) & $3591.2 \pm 937.8$ & $3282.1 \pm 995.2$ & 0.028 \\
\hline in/out balance (ml) & $1826 \pm 1168.9$ & $1573.7 \pm 888.1$ & 0.112 \\
\hline maximum heart rate in AF (bpm) & $94.8 \pm 18.4$ & $105 \pm 24.9$ & $<0.001$ \\
\hline maximum systolic blood pressure (mmHg) & $144.8 \pm 23.0$ & $144.4 \pm 23.1$ & 0.91 \\
\hline with/without PVI & $20 / 42$ & $100 / 212$ & 0.975 \\
\hline
\end{tabular}

AF: Atrial fibrillation; TIA: Transient ischemic attack; CAVI: Cardio-ankle vascular index; baPWV: Brachial-ankle pulse wave velocity; eGFR: Estimated glomerular filtration rate; BNP: B-type natriuretic peptide; NT-proBNP: N-terminal pro-B-type natriuretic peptide; CRP: C-reactive protein; HbA1c: Hemoglobin A1c; LA: Left atrial; LAVI: Left atrial volume index; LV: Left ventricular; TR: Tricuspid valve regurgitation; RVSP: Right ventricular systolic pressure; IVC: Inferior vena cava; TEE: Transesophageal echocardiography; ACEI: Angiotensin-converting enzyme inhibitor; ARB: Angiotensin receptor blocker; PVI: Pulmonary vein isolation.

\section{Results}

The patients characteristics and procedural data are shown in Table 1 . We retrospectively analyzed the data the day after ablation. According to RVSP the day after ablation, we divided the patients into two groups as follows: high RVSP group (RVSP $\geq 40 \mathrm{mmHg}$ the day after ablation, $\mathrm{n}=62$ ) and normal RVSP group (RVSP < $40 \mathrm{mmHg}$ the day after ablation, $\mathrm{n}=312$ ).

\section{Univariate analysis}

Comparison of characteristics of patients between the normal and high RVSP groups is shown in Table 1. Age was older in the high RVSP group than in the normal RVSP group $(p<0.001)$. The prevalence of paroxysmal AF was greater in the high RVSP group than in the normal RVSP group $(p=0.025)$. CHADS2 score $(p<$ $0.001)$ and CHA2DS2-VASc score $(p<0.001)$ were higher in the high RVSP group than in the normal RVSP group. In the components of CHADS2 score, age $\geq 65$-yearsold $(p=0.002)$, history of congestive heart failure ( $p$ $=0.045)$, hypertension $(p=0.011)$, diabetes mellitus $(<0.001)$, stroke/transient ischemic attack $(p=0.034)$ were higher in the high RVSP group than in the normal RVSP group. Mean cardio-ankle vascular index $(p=$ 0.002 ) and mean brachial-ankle pulse wave velocity ( $p$ $=0.001$ ) were higher in the high RVSP group than in the normal RVSP group. In the blood sample examination before procedure, serum creatinine concentrations $(p=$ $0.012)$, estimated glomerular filtration rate $(p=0.001)$, B-type natriuretic peptide levels $(p=0.009), N$-terminal pro-B-type natriuretic peptide levels $(p=0.017)$, and hemoglobin A1c levels $(p=0.006)$ were higher in the high RVSP group than in the normal RVSP group. In the echocardiographic data before procedure, LA dimension $(p=0.001)$, LA volume $(p=0.022)$, LA volume index $(p=0.024)$, mitral inflow $E$ wave velocity $(p=0.039)$, peak flow velocity of tricuspid valve regurgitation ( $p$ $<0.001)$, RVSP $(p=0.004)$, tissue Doppler septal $e^{\prime}$ wave velocity $(p=0.006)$, E/e' septal ratio $(p=0.003)$ were higher in the high RVSP group than in the normal RVSP group. LA appendage flow velocity calculated by transesophageal echocardiography was higher in the high RVSP group than in the normal RVSP group ( $p=$ 0.046). The prevalence of the patients who prescribed angiotensin-converting enzyme inhibitors/angiotensin receptor blockers $(p=0.012)$ and diuretics $(p=0.022)$ was higher in the high RVSP group than in the normal RVSP group, and who prescribed class 1 antiarrhythmic drugs ( $p=0.043$ ) was less in the high RVSP group than in the normal RVSP group. In the data during procedure, total amount of injection during the procedure was greater in the high RVSP group than in the normal RVSP group ( $p=0.028)$, and maximum heart rate of AF during the procedure less in the high RVSP group than in the normal RVSP group $(p<0.001)$. Although, there was no significant difference in sex, body mass index, or left ventricular ejection fraction between the two groups.

\section{Multivariate analysis for high RVSP}

There were many predictors in multivariate analysis in this study. Then we selected predictors using in multivariate analysis considering the confounding factors as follows. In multivariate analysis, age $>65$-years-old (OR 2.62; 95\% confidence interval 1.37-5.09; $p=0.004$ ), history of diabetes mellitus (OR 6.7; 95\% confidence interval 2.83-16.00; $\mathrm{p}<0.001$ ), LA dimension (OR 1.06; 95\% confidence interval 1.01-1.13; $p=0.021$ ), and mitral inflow E wave (OR 1.02; 95\% confidence interval 1.001.03; $p=0.046$ ) were predictors of high RVSP the day after ablation (Table 2).

\section{Discussion}

We investigated patients who underwent AF ablation using an irrigation catheter. Until irrigation catheter was available, $8 \mathrm{~mm}$ tip catheter had been standard for AF ablation. But irrigation catheter replaced rapidly because of the reduction of the complication of thromboembolic events. But the volume overload in AF ablation with irrigation catheter was not neglectable. More than 
Table 2: Multivariate analysis of predictors of high right ventricular systolic pressure.

\begin{tabular}{|l|l|l|l|}
\hline & odds ratio & Cl (95\%) & P value \\
\hline Age $\geq$ 65-years-old & 2.62 & $1.37-5.09$ & 0.004 \\
\hline mean of both CAVI & 1.15 & $0.89-1.50$ & 0.295 \\
\hline paroxysmal/persistent AF & 1.22 & $0.58-2.56$ & 0.591 \\
\hline creatinine & 1.93 & $0.69-5.52$ & 0.192 \\
\hline total injection during procedure & 1 & $0.99-1.00$ & 0.207 \\
\hline LA dimension before ablation & 1.06 & $1.01-1.13$ & 0.021 \\
\hline mitral inflow E wave (cm/sec) & 1.02 & $1.00-1.03$ & 0.049 \\
\hline diabetes mellitus & 6.7 & $2.83-16.00$ & $<0.001$ \\
\hline congestive heart failure [n (\%)] & 1.71 & $0.63-4.26$ & 0.263 \\
\hline
\end{tabular}

CAVI: Cardio ankle vascular index; AF: Atrial fibrillation; LA: Left atrial.

3 liters of injection volume were used in the procedure with the NaviStar ThermoCool catheter. There have been few reports of volume overload in clinical practice $[18,19]$.

In general, left ventricular systolic or diastolic dysfunction is the main reason of heart failure. Then the main reasons of heart failure caused by volume overload are suspected to be lower ejection fraction or diastolic dysfunction estimated by echocardiography. Although ejection fraction was not a predictor of high RVSP after catheter ablation in our data. And the parameters of diastolic left ventricular dysfunction, such as mitral inflow $E$ wave velocity was higher in the high RSVP group, but age or the history of diabetes mellitus is stronger predictors of higher RVSP after catheter ablation using multivariate analysis. Then we may need close observation to the patients of high age and/or who have a history of diabetes, when not only in the patients undergo catheter ablation but also other operations with volume overload. Consideration of age and a history of diabetes mellitus might be helpful to avoid heart failure in the perioperative period.

\section{Study Limitations}

There are several limitations to this study. First, this study was retrospectively analyzed from a single center. Therefore, a further prospective, large study is required to confirm our results. Second, the high RVSP group included patients with a higher RVSP and a history of congestive heart failure. There could have been some bias, although a history of congestive heart failure was not a predictor of high RVSP in multiple regression analysis.

\section{Conclusions}

Close observation is important with an older age, a history of diabetes mellitus, and a greater LA dimension and/or mitral $E$ wave to avoid heart failure after $A F$ ablation using irrigation catheters.

\section{Funding Sources}

This work was supported by the Japan Society for the Promotion of Science (JSPS) KAKENHI (Grant Number
26461077).

\section{Acknowledgment}

We thank Ellen Knapp, PhD, from Edanz Group (www.edanzediting.com/ac) for editing a draft of this manuscript.

\section{Conflicts of Interest}

There are no conflicts of interest for this study.

\section{References}

1. Haïssaguerre $M$, Jaïs $P$, Shah $D C$, Takahashi $A$, Hocini $M$, et al. (1998) Spontaneous initiation of atrial fibrillation by ectopic beats originating in the pulmonary veins. $\mathrm{N}$ Engl $\mathrm{J}$ Med 339: 659-666.

2. Wazni OM, Marrouche NF, Martin DO, Verma A, Bhargava $\mathrm{M}$, et al. (2005) Radiofrequency ablation vs antiarrhythmic drugs as first-line treatment of symptomatic atrial fibrillation: A randomized trial. JAMA 293: 2634-2640.

3. Pappone C, Rosanio S, Augello G, Gallus G, Vicedomini $\mathrm{G}$, et al. (2003) Mortality, morbidity, and quality of life after circumferential pulmonary vein ablation for atrial fibrillation: outcomes from a controlled nonrandomized long-term study. J Am Coll Cardiol 42: 185-197.

4. Cappato R, Calkins H, Chen SA, Davies W, lesaka Y, et al. (2005) Worldwide survey on the methods, efficacy, and safety of catheter ablation for human atrial fibrillation. Circulation 111: 1100-1105.

5. Stabile G, Bertaglia E, Senatore G, De Simone A, Zoppo $F$, et al. (2006) Catheter ablation treatment in patients with drug-refractory atrial fibrillation: a prospective, multi-centre, randomized, controlled study (Catheter ablation for the cure of atrial fibrillation study). Eur Heart J 27: 216-221.

6. Oral H, Chugh A, Good E, Wimmer A, Dey S, et al. (2007) Radiofrequency catheter ablation of chronic atrial fibrillation guided by complex electrograms. Circulation 115: 26062612.

7. Nademanee K, McKenzie J, Kosar E, Schwab M, Sunsaneewitayakul B, et al. (2004) A new approach for catheter ablation of atrial fibrillation: Mapping of electrophysiologic substrate. J Am Coll Cardiol 43: 20442053.

8. Senni M, Tribouilloy CM, Rodeheffer RJ, Jacobsen SJ, Evans JM, et al. (1998) Congestive heart failure in the community: A study of all incident cases in Olmsted County, Minnesota, in 1991. Circulation 98: 2282-2289. 
9. Zile VIR, Baicu CF, Gaasch WH (2004) Diastolic heart failure abnormalities in active relaxation and passive stiffness of the left ventricle. N Engl J Med 350: 1953-1959.

10. Gandhi SK, Powers JC, Nomeir AM, Fowle K, Kitzman DW, et al. (2001) The pathogenesis of acute pulmonary edema associated with hypertension. N Engl J Med 344: 17-22.

11. Calkins H, Brugada J, Packer DL, Cappato R, Chen SA, et al. (2007) HRS/EHRA/ECAS expert Consensus Statement on catheter and surgical ablation of atrial fibrillation: recommendations for personnel, policy, procedures and follow-up. A report of the Heart Rhythm Society (HRS) Task Force on catheter and surgical ablation of atrial fibrillation. Heart Rhythm 4: 816-861.

12. Nademanee K, Schwab MC, Kosar EM, Karwecki M, Moran MD, et al. (2008) Clinical outcomes of catheter substrate ablation for high-risk patients with atrial fibrillation. J Am Coll Cardiol 51: 843-849.

13. Nademanee K, Lockwood E, Oketani N, Gidney B (2010) Catheter ablation of atrial fibrillation guided by complex fractionated atrial electrogram mapping of atrial fibrillation substrate. J Cardiol 55: 1-12.

14. Iriki Y, Ishida S, Oketani N, Ichiki H, Okui H, et al. (2011) Relationship between clinical outcomes and unintentional pulmonary vein isolation during substrate ablation of atrial fibrillation guided solely by complex fractionated atrial electrogram mapping. J Cardiol 58: 278-286.
15. Ichiki H, Oketani N, Ishida S, Iriki Y, Okui H, et al. (20120) Incidence of asymptomatic cerebral microthromboembolism after atrial fibrillation ablation guided by complex fractionated atrial electrogram. J Cardiovasc Electrophysiol 23: 567-573.

16. Maenosono R, Oketani N, Ishida S, Iriki Y, Ichiki H, et al. (2012) Effectiveness of esophagus detection by three-dimensional electroanatomical mapping to avoid esophageal injury during ablation of atrial fibrillation. J Cardiol 60: 119125.

17. Ichiki H, Oketani N, Ishida S, Iriki Y, Okui H, et al. (2013) The incidence of asymptomatic cerebral microthromboembolism after atrial fibrillation ablation: Comparison of warfarin and dabigatran. Pacing Clin Electrophysiol 36: 1328-1335.

18. Seiler J, Steven D, Roberts-Thomson KC, Inada K, Tedrow UB, et al. (2014) The effect of open-irrigated radiofrequency catheter ablation of atrial fibrillation on left atrial pressure and B-type natriuretic peptide. Pacing Clin Electrophysiol 37: $616-623$

19. Nölker G, Gutleben KJ, Ritscher G, Rittger H, Asbach S, et al. (2011) Left atrial lesion formation and volume overload by open irrigation ablation technology during pulmonary vein antrum isolation: acute effects on cardiac hemodynamics. J Interv Card Electrophysiol 31: 125-230. 